They worked out that telomerase provides a platform enabling DNA polymerases to copy the entire length of the chromosome without missing the ends. Greider and Blackburn also showed that telomerase contains a key RNA sequence that acts as a template for the telomere $\mathrm{DNA}^{3}$, which attracts proteins to form a protective cap around the ends of the DNA strands.

Telomeres themselves shorten with repeated cell division, making up a key part of the cell's ageing mechanism. Low telomerase activity and telomere shortening speed up ageing, whereas incessantly dividing cancer cells often have high telomerase activity and maintain their telomere length. Cancer therapies directed against telomerase are now being tested in clinical trials.

But there is still a lot of basic biology to discover - such as how telomerase activity is regulated at individual telomeres, and how telomeres manage to avoid the attentions of DNA repair enzymes which seek out breaks in DNA and restitch the torn ends.

Blackburn and Greider become only the ninth and tenth female scientists to win the physiology or medicine prize since it was first awarded in 1901, and it is the first time that two women have been recognized in a single prize. Indeed, telomere research is unusually dominated by women. "It is hard to find a male among us," says David Shore, a cell biologist at the University of Geneva, Switzerland. "And two main reasons are Liz and Carol - they created the field and have been role models."

Blackburn has also been involved in the politics of science, serving on the US President's Council on Bioethics from 2002 until she was dropped in 2004 after criticizing the restrictions on human embryonic stem-cell research imposed by then President George W. Bush.

Lea Harrington, Greider's first graduate student, who is now at the Wellcome Trust Centre for Cell Biology at the University of Edinburgh, UK, says that her four years in Greider's laboratory at Cold Spring Harbor, New York, were "electric. We all realized what an exciting time it was - so many questions being answered about the composition of telomerase, how it worked and its relevance to human biology."

Alison Abbott

1. Szostak, J. W. \& Blackburn, E. H. Cell 29, 245-255 (1982).

2. Greider, C. W. \& Blackburn, E. H. Cell $\mathbf{4 3}, 405-413$ (1985)

3. Greider, C. W. \& Blackburn, E. H. Nature 337, 331-337 (1989).

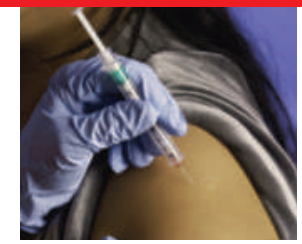

FUTURE OF HIV VACCINE UNCLEAR

Puzzles to solve before trials can move forward. www.nature.com/news

\title{
Nobel Prize in Physics awarded to light pioneers
}

Two technologies that revolutionized science, computing and communication have secured their developers a share of the Nobel Prize in Physics.

Charles Kao of the Chinese University of Hong Kong has won half the prize for his role in developing fibre-optic cables. The other half is shared by Willard Boyle and George Smith of Bell Laboratories in Murray Hill, New Jersey, for their development of the charge-coupled device (CCD), an electronic chip that converts light into a digital signal.

In 1969, Boyle and Smith developed a chip that could transform light into an electronic signal. The duo used newly discovered metal oxide semiconductors that could convert photons into a flow of electrons, which could be read from the edges of the chip and used to recreate the image. The ability to digitally capture light has found application in nearly every field of science - particularly astronomy. "Basically, they revolutionized optical astronomy," says Mark Casali, head of instrumentation at the European Southern Observatory in Garching, Germany. Before the advent of CCDs, astronomers were imaging stars using photographic plates, which were less sensitive and less precise than their digital successors, Casali says. Using CCD cameras, astronomers have been able to discover faint galaxies and even see fluctuations in a star's

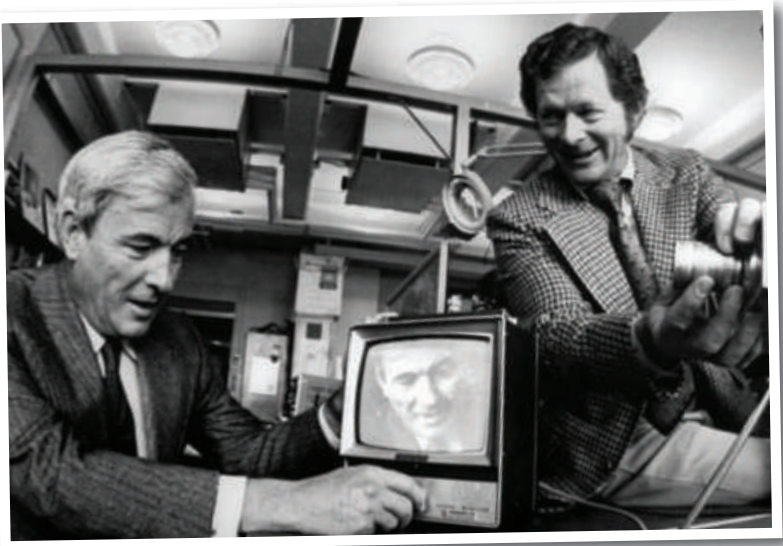

Willard Boyle and George Smith invented charge-coupled devices. light created by an orbiting planet.

The detectors also made space-based astronomy a reality, says Matt Mountain, director of the Space Telescope Science Institute in Baltimore, Maryland, which coordinates science for the Hubble Space Telescope. "It made telescopes like the Hubble possible," he says. "You could now put large electronic detectors in space that could beam down digital pictures of some of the faintest objects human beings have ever seen."

Fibre optics has had an equally impressive impact on science, not least by facilitating collaboration on a global scale. But the transmission of data over thousands of kilometres seemed a distant dream when Kao first began his work on fibre-optic cables. Back then, fibres could carry light only a few metres by total internal reflection before the signal faded. Kao and his colleagues at Standard Telecommunication Laboratories in Harlow, UK, worked out that impurities, mainly iron ions, were causing the loss. Kao identified an alternative material - fused silica that could carry light over much greater distances without significant loss. The work ultimately led to the billion-kilometre-long network of fibre-optic cables that span the globe today.

Fibre optics will also have a pivotal role in the world's largest science experiment, the Large Hadron Collider (LHC) at CERN, Europe's particle-physics centre near Geneva, Switzerland. The LHC's largest detectors create around a million gigabytes of raw data every second. The cables then shepherd the data to nearby servers and on to thousands of scientists in 33 countries through an ultrafast computer grid. "The whole infrastructure is based on optical fibre," says Ian Bird, the grid's project leader. "There's no way that our data rates could be sustained without it." Geoff Brumfiel 